\title{
Conocimiento de la urticaria crónica entre médicos especialistas y residentes de dermatología en Colombia: estudio transversal basado en una encuesta a un grupo de médicos
}

Knowledge of the chronic urticaria between specialist physicians and residents of dermatology in Colombia: crosssectional study based on survey to a sample of physicians

\section{Natalia Hernández ${ }^{1}$, Diana Carolina Tamayo²}

1. Médica, especialista en Dermatología, Alergias Cutáneas y Dermatología genital. Dermatología Ocupacional. Magíster en Economía de la Salud y del Medicamento

2. Médica, magíster en Epidemiología Clínica. Médica de enlace científico en dermatología, para Novartis de Colombia

\section{RESUMEN}

La urticaria crónica (UC) se define como una condición caracterizada por la aparición de habones, angioedema o ambos, durante un lapso mayor de 6 semanas. Esta investigación tuvo como objetivo identificar el grado de conocimiento sobre UC entre médicos especialistas y residentes de dermatología en Colombia, a través de una encuesta realizada entre 86 profesionales sanitarios. Más del $90 \%$ de los participantes coincidió con la definición correcta de UC. Cerca del $50 \%$ de los especialistas y dos tercios de los residentes consideraron el uso de diversos exámenes paraclínicos como parte del ejercicio diagnóstico, mientras que la mayoría no contempló el uso rutinario de la biopsia. La mayoría de los participantes reconoció el impacto que la enfermedad tiene sobre la calidad de vida. El 50 \% de los especialistas no utiliza instrumentos de clinimetría y el $50 \%$ de los residentes no los conocen. Este es el primer estudio que explora el grado de conocimiento de UC entre los dermatólogos en Colombia y expone oportunidades de mejoramiento en la práctica clínica, de acuerdo con las recomendaciones de las guías internacionales vigentes.

PALABRAS CLAVE: urticaria, Colombia, encuesta.

\section{SUMMARY}

Chronic urticaria (CU) is defined as a condition characterized by the appearance of wheals, angioedema or both, for a period greater than 6 weeks. The objective of this research was to identify the degree of knowledge about UC among medical specialists and residents of dermatology in Colombia, through a survey of 86 physicians. More than $90 \%$ of the participants agreed with the proper definition of UC. Nearly $50 \%$ of the specialists and two-thirds of residents consider the use of various tests as part of the diagnostic exercise, while
Correspondencia:

Natalia Hernández

Email:

nataliahernandez67@gmail. com

Recibido: 02/09/18

Aceptado: 18/o3/19

Conflictos de interés:

No se reportan conflictos de interés.

Financiación:

Ninguna. 
most do not consider the routine use of biopsy. The impact of the disease on the quality of life is recognized by most of the participants. $50 \%$ of the specialists do not use clinimetry tools and $50 \%$ of the residents do not know them. This is the first study that explores the knowledge of UC among dermatologists in Colombia and exposes opportunities for improvement in clinical practice in accordance with the recommendations of the current international guidelines.

KEY WORDS: Urticaria, Colombia, surveys and questionnaires.

\section{INTRODUCCIÓN}

La urticaria se define como una condición caracterizada por la aparición de habones, angioedema o $\operatorname{ambos}^{(1)}$. De acuerdo con las más recientes guías internacionales de práctica clínica, se recomienda clasificar la urticaria, según su duración, en aguda (menor o igual a 6 semanas) o crónica (UC) (mayor de 6 semanas) ${ }^{(1,2)}$. Con base en su naturaleza, se clasifica como espontánea o inducible, según se dé la presencia de un factor desencadenante específico o no ${ }^{(1,2)}$. La forma espontánea afecta entre el 0,5 \% y el 1 \% de la población ${ }^{(3)}$.

En Colombia, hace dos décadas, dos instituciones hospitalarias de Medellín determinaron que la urticaria correspondía a un o,8 \% de las consultas dermatológicas ${ }^{(4)}$. En un reciente estudio realizado en Italia, Rimoldi, et al., establecieron que entre especialistas en dermatología y alergología se atendía una mediana de 40 pacientes con urticaria crónica espontánea (UCE) anualmente, con una incidencia de angioedema del $35,9 \%{ }^{(3)}$. Esta investigación tiene como objetivo identificar, mediante una encuesta, el grado de conocimiento sobre UC y UCE entre médicos especialistas y residentes de dermatología en Colombia.

\section{MÉTODOS}

Este estudio responde a un diseño observacional, de curso transversal. Se condujo una encuesta dirigida a profesionales sanitarios, para lo que se formuló un cuestionario que indagara los aspectos generales de la práctica profesional en dermatología y los aspectos relevantes sobre la epidemiología, las manifestaciones y categorización clínicas, los factores asociados, la clinimetría y la aproximación terapéutica en UC. La elaboración del cuestionario se hizo con base en los referentes citados en la literatura reciente, y su diseño, de autoría del grupo investigador (anexo 1), consistió en 17 preguntas de selección múltiple, en idioma castellano, como parte de una encuesta anónima y en formato impreso. De acuerdo con los objetivos planteados, se tuvieron en cuenta los siguientes criterios para la selección de los participantes para responder la encuesta: médicos dermatólogos, médicos residentes de dermatología, ejercicio profesional en Colombia y aceptación voluntaria para responder a la encuesta. La selección de los participantes se dio a través del muestreo por conveniencia, después de la convocatoria llevada a cabo en dos eventos académicos de carácter nacional, dirigidos justamente a profesionales de la dermatología, realizados en 2017.

Una vez recolectados los formatos de la encuesta, se diseñó y diligenció una base de almacenamiento de datos en Excel. También se elaboraron unas tablas de frecuencias absoluta y relativa para cada una de las variables. Los resultados se presentan en términos de proporciones, dado que las variables se recopilaron en escalas nominal y categórica.

\section{RESULTADOS}

Participaron 54 especialistas y 32 residentes de dermatología en Colombia, procedentes de diferentes ciudades del país.

Entre el grupo de especialistas, el perfil de experiencia laboral como dermatólogo identificado correspondió a:

- $\quad$ Menos de 5 años: $29,6 \%$

- Entre 5 y 10 años: $18,5 \%$

- Entre 10 y 15 años: 25,9\%

- Más de 15 años: 24,1\%

A su vez, el perfil de residentes participantes correspondió a:

- $\quad$ Primer año: 40,6\%

- Segundo año: $37,5 \%$

- $\quad$ Tercer año 21,9\%

Sobre el sitio de práctica clínica habitual, tanto especialistas como residentes manifestaron desarrollar sus actividades en diferentes tipos de instituciones (tabla 1). 


\section{Conocimiento sobre clínica y diagnóstico de la urticaria crónica}

Respecto del conocimiento sobre la definición vigente de carácter crónico de la urticaria, el 92,6 \% de los especialistas y el 96,9\% de los residentes reconocieron el punto de corte de 6 semanas como el tiempo de evolución que determina como crónico el curso de esta enfermedad.

Sobre la noción de la prevalencia de la UC entre la población general, el 44,4 \% de los especialistas y el 40,0 $\%$ de los residentes expresaron que corresponde a un 2 \%-3 \%. En correspondencia, la mayoría de los participantes señaló que la frecuencia de UC en su consulta es inferior al $5 \%$ (tabla 2). Dentro de ese perfil de pacientes, de acuerdo con lo reportado por los médicos, la frecuencia de UCE en su consulta es variable (tabla 3).

En cuanto a la coexistencia de angioedema, el 66,7 \% de los especialistas y el 53,1 \% de los residentes manifestaron conocer que puede ocurrir en más del $50 \%$ de los pacientes con UCE.
En lo que respecta a la solicitud de exámenes paraclínicos como parte del estudio diagnóstico de la UC, un gran porcentaje de los especialistas y los residentes manifestó que suelen recurrir a hemogramas, pruebas microbiológicas del tracto gastrointestinal y pruebas funcionales tiroideas, entre otras (tabla 4). Sobre el uso rutinario de la biopsia, la mayoría de los encuestados expresó no estar a favor de esta práctica: 85,2 \% de los especialistas y 90,6 \% de los residentes. En relación con la indicación de pruebas de provocación, el 46,3\% de los especialistas y el 59,4 \% de los residentes se mostraron a favor de este recurso dentro del ejercicio diagnóstico en UC.

\section{Evaluación de la actividad y el impacto de la enfermedad en la calidad de vida}

Mediante el cuestionario también se indagó acerca del conocimiento y el uso de instrumentos de clinimetría en UC entre los profesionales sanitarios encuestados.

Tabla 1. Distribución de los participantes de acuerdo con el sitio de su práctica clínica

\begin{tabular}{|c|c|c|}
\hline Tipo de institución & Especialista (\%) & Residente (\%) \\
\hline Hospital universitario & 14,8 & 68,8 \\
\hline Consultorio privado & 14,8 & 3,1 \\
\hline $\begin{array}{l}\text { Consulta institucional } \\
\text { para aseguradores }\end{array}$ & 5,6 & - \\
\hline Varios de los anteriores & 64,8 & 25 \\
\hline
\end{tabular}

Tabla 2. Distribución de los participantes de acuerdo con la frecuencia de urticaria crónica entre los pacientes que atienden en su consulta

\begin{tabular}{lcc}
$\begin{array}{l}\text { Frecuencia de urticaria crónica } \\
\text { en la consulta }\end{array}$ & Especialista (\%) & Residente (\%) \\
\hline Menos del $5 \%$ de los pacientes & 79,6 & 65,6 \\
\hline $5 \%-10 \%$ de los pacientes & 14,8 & 25 \\
Más del $10 \%$ de los pacientes & 5,6 & 6,3
\end{tabular}


Tabla 3. Distribución de los participantes de acuerdo con la frecuencia de urticaria crónica espontánea, dentro del universo de pacientes con urticaria cónica que atienden en su consulta

\begin{tabular}{lcc}
$\begin{array}{l}\text { Frecuencia de urticaria crónica } \\
\text { espontánea en la consulta }\end{array}$ & Especialista (\%) & Residente (\%) \\
\hline Más del $50 \%$ de los pacientes con UC & 44,4 & 34,4 \\
\hline $20 \%-50 \%$ de los pacientes con UC & 14,8 & 37,5 \\
\hline Menos del $20 \%$ de los pacientes con UC & 37 & 28,1
\end{tabular}

Tabla 4. Distribución de los participantes de acuerdo con la preferencia de estudios paraclínicos dentro del diagnóstico de urticaria crónica

\begin{tabular}{lcc}
$\begin{array}{l}\text { Estudios } \\
\text { solicitados }\end{array}$ & paraclínicos & Residente (\%) \\
$\begin{array}{l}\text { Ninguno } \\
\begin{array}{l}\text { Cuadro hemático + pruebas funcionales } \\
\text { tiroideas + coprológico }\end{array}\end{array}$ & 5,6 & 6,3 \\
$\begin{array}{l}\text { Cuadro hemático + pruebas funcionales } \\
\text { tiroideas + prueba de hepatitis + } \\
\text { búsqueda de Helicobacter pylori }\end{array}$ & 42,6 & 15,6 \\
$\begin{array}{l}\text { Cuadro hemático + pruebas funcionales } \\
\text { tiroideas + inmunoglobulina E }\end{array}$ & 33,3 & 59,4 \\
\hline
\end{tabular}

En cuanto al conocimiento de la UAS7 (escala de actividad de la urticaria durante 7 días, por sus siglas en inglés) y de instrumentos de este tipo, los resultados mostraron que el $50 \%$ de los especialistas los conocen, pero no los utilizan rutinariamente, mientras que el 50 $\%$ de los residentes manifestó no conocerlos (figura 1). Con respecto a la percepción del impacto de la UC sobre la calidad de vida de los pacientes, el 96,3\% de los especialistas y el $87,5 \%$ de los residentes consideraron que la calidad del sueño y la productividad laboral se ven afectadas significativamente. Sin embargo, en relación con el conocimiento y la implementación de escalas de evaluación de la calidad de vida en UC, aproximadamente el $50 \%$ de especialistas y de residentes manifestaron conocer los instrumentos, pero no usarlos de rutina.

\section{DISCUSIÓN}

Esta encuesta identificó un nivel relevante de conocimiento sobre los conceptos básicos relacionados con la UC. La gran mayoría de los participantes conoce el lapso que define la cronicidad y es consciente de la prevalencia de angioedema en esta condición.

Los estudios desarrollados previamente en el contexto regional indican que, hasta hace dos décadas, la urticaria correspondía a menos del $1 \%$ de las consultas dermatológicas (4), según los registros formales de diagnóstico. En el escenario actual, en el que la prevalencia conocida de la UCE está alrededor del $1 \%$-2 \% en la población general y del $5 \%$ para cualquier forma de urticaria ${ }^{(5-7)}$, más del $20 \%$ de los especialistas y del 30 $\%$ de los residentes encuestados manifestaron que la 


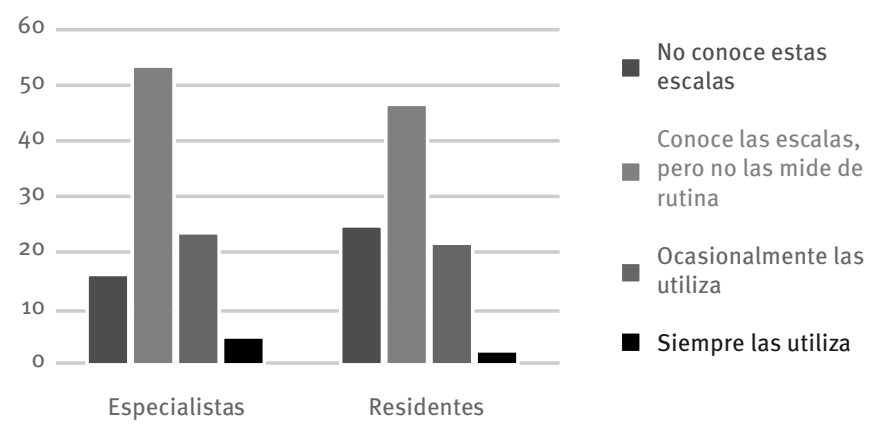

Figura 1. Distribución de los participantes de acuerdo con el conocimiento y el uso de instrumentos de clinimetría tipo UAS7.

frecuencia es mayor al $5 \%$ de sus consultas, lo que podría deberse a que entre médicos y pacientes hay mayor conciencia de la enfermedad y a que la demanda de atención por urticaria es creciente. Se identifica así una necesidad de incrementar la sensibilidad local frente al conocimiento y la detección de la UCE, dado que la prevalencia global es de dos tercios de la UC ${ }^{(5-7)}$, mientras que los médicos encuestados la consideran menor. En cuanto a los diferentes aspectos del diagnóstico y manejo de la UC y la UCE, la encuesta expuso varias oportunidades de enfatizar recomendaciones basadas en la evidencia y de buena práctica clínica. En el contexto latinoamericano, un reciente estudio encontró que el 19,8 \% de los médicos tratantes de urticaria tenía conciencia sobre la existencia de las guías vigentes ${ }^{(8)}$. Se identificó una tendencia relevante, tanto entre especialistas como en residentes, hacia la solicitud de exámenes paraclínicos en conjunto, de manera rutinaria, en el abordaje diagnóstico de la UC. De acuerdo con las versiones más recientes de las guías internacionales de UC ${ }^{(1,2)}$, se recomienda limitar el uso de exámenes diagnósticos de rutina y favorecer la orientación según los factores desencadenantes aparentes que se identifiquen en la historia clínica. Las guías más recientes (2) recomiendan como pruebas habituales el cuadro hemático y la proteína C-reactiva o la velocidad de sedimentación globular, mientras que los estudios de extensión deben reservarse para los casos cuya orientación clínica lo señale o en los casos de larga duración o de difícil control.

Una de las grandes oportunidades para afianzar el conocimiento y la implementación de los estándares de práctica clínica se da en el escenario del uso e interpretación de los instrumentos de clinimetría. Alrededor del $50 \%$ de los encuestados manifestaron tener co- nocimiento de tales instrumentos. De acuerdo con las recomendaciones vigentes, en cada consulta se deben evaluar tres aspectos importantes de la UC: actividad de la enfermedad, control de la enfermedad e impacto sobre la calidad de vida (2). Tales referentes sugieren el uso de escalas propias para la UC y que faciliten la toma de decisiones terapéuticas. Para evaluar la actividad de la enfermedad, se sugiere el uso de la UAS7; para evaluar el control de la enfermedad, se sugiere el uso de la UCT (prueba de control de la urticaria, por sus siglas en inglés); y para evaluar el impacto de la UC sobre la calidad de vida, se sugiere el uso del CU-Q20L (cuestionario de calidad de vida en urticaria crónica, por sus siglas en inglés); todos estos instrumentos cuentan con versiones validadas en castellano ${ }^{(2,9-11)}$. Incluso, en algunos estudios sobre intervenciones terapéuticas en UC, se utiliza también el DLQI (índice de calidad de vida en dermatología, por sus siglas en inglés) para la valoración de la calidad de vida de los pacientes ${ }^{(2-15)}$. El uso sistemático de instrumentos de clinimetría facilita el seguimiento y la toma de decisiones en el escenario clínico, así como el desarrollo de proyectos de investigación. Es imperativo afianzar la exploración, el entendimiento y la intervención sobre el impacto de esta condición en la calidad de vida de los pacientes, sobre todo cuando es conocido que, en Colombia, la urticaria hace parte de las enfermedades dermatológicas que más afectan la calidad de vida, en especial la dimensión emocional, con índices de afectación comparables a los de la psoriasis, la dermatitis de contacto, la dermatitis atópica y la condilomatosis, tal como lo demostró un reciente estudio en una cohorte de pacientes de diversas regiones del país ${ }^{(16)}$. 


\section{CONCLUSIÓN}

Los resultados de este estudio son relevantes debido a que proceden de una encuesta realizada directamente a los dermatólogos en Colombia, que permite conocer los comportamientos de residentes y especialistas en relación con la urticaria crónica (UC). Los dermatólogos graduados o en formación conocen la enfermedad y su contundente impacto en la calidad de vida. La solicitud de exámenes paraclínicos es una práctica frecuente, aunque las últimas guías indican que esta debe estar orientada por la historia clínica. Es importante insistir en la relevancia del uso de la clinimetría para el diagnóstico y el seguimiento de la enfermedad, lo cual, como es sugerido en las guías internacionales, debe ser rutinario.

\section{REFERENCIAS}

1. Zuberbier T, Aberer W, Asero R, Abdul Latiff AH, Baker D, Ballmer-Weber B, et al. The EAACI/ $\mathrm{GA}^{2} \mathrm{LEN} / \mathrm{EDF} / \mathrm{WAO}$ guideline for the definition, classification, diagnosis and management of urticaria. Allergy. 2018;73(7):1393-414. doi: 10.1111/ all.13397.

2. Zuberbier T, Aberer W, Asero R, Bindslev-Jensen C, Brzoza Z, Canonica GW, et al. The EAACI/ GA(2) LEN/EDF/WAO Guideline for the definition, classification, diagnosis, and management of urticaria: the 2013 revision and update. Allergy. 2014;69(7):868-87. doi: 10.1111/all.12313.

3. Rimoldi M, Rossi O, Rota N. State of the art of chronic spontaneous urticaria in Italy: a multicentre survey to evaluate physicians' and patients' perspectives. BMJ Open. 2016;6(10):eo12378. doi: 10.1136/bmjopen-2016-012378.

4. Sanclemente G, Mahecha M, Guzmán C. Enfermedades de la piel más frecuentes en la consulta externa dermatológica del Hospital Universitario San Vicente de Paúl y del Hospital Infantil. Medellín, 1999. Acta Med Colomb. 2001;26(5):240-44.

5. Gaig P, Olona M, Muñoz Lejarazu D, Caballero MT, Domínguez FJ, Echechipia S, et al. Epidemiology of urticaria in Spain. J Investig Allergol Clin Immunol. 2004;14(3):214-20.

6. Zuberbier T, Balke M, Worm M, Edenharter G, Maurer M. Epidemiology of urticaria: a representative cross-sectional population survey. Clin Exp Dermatol. 2010;35(8):869-73. doi: 10.1111/j.13652230.2010.03840.x.

7. Furue M, Yamazaki S, Jimbow K, Tsuchida T, Amagai M, Tanaka T, et al. Prevalence of dermatological disorders in Japan: a nationwide, cross-sectional, seasonal, multicenter, hospitalbased study. J Dermatol. 2011;38(4):310-20. doi: 10.1111/j.1346-8138.2011.01209.x.

8. Calderón JC, Chérrez Ojeda I, Weller K, Maurer M, Chérrez A. Management of chronic urticaria in real life: cross-sectional physical-based survey study in Latin-America. Allergy. 2016;(Suppl. 102):52891. Poster 1253.

9. Valero A, Herdman M, Bartra J, Ferrer M, Jáuregui I, Dávila I, et al. Adaptation and validation of the Spanish version of the Chronic Urticaria Quality of Life Questionnaire (CU-Q2oL). J Investig Allergol Clin Immunol. 2008;18(6):426-32.

10. Balañá M, Valero A, Giménez Arnau A, Ferrer $\mathrm{M}$, Jauregui I, Ballesteros C, et al. Validation of The Spanish Version of The Urticaria Activity Score (Uas) and Its Use Over One Week (Uas7). Value Health. 2015;18(7):A426. doi: 10.1016/j. jval.2015.09.584.

11. García-Díez I, Curto-Barredo L, Weller K, Pujol RM, Maurer M, Giménez-Arnau AM. Cross-Cultural Adaptation of the Urticaria Control Test From German to Castilian Spanish. Actas Dermosifiliogr. 2015;106(9):746-52. doi: 10.1016/j.ad.2015.05.009.

12. Maurer M, Staubach P, Raap U, Richter-Huhn G, Bauer A, Ruëff F, et al. H1-antihistamine-refractory chronic spontaneous urticaria: it's worse than we thought - first results of the multicenter real-life AWARE study. Clin Exp Allergy. 2017;47(5):684-92. doi: 10.1111/cea.12900.

13. Saini SS, Bindslev-Jensen C, Maurer M, Grob JJ, Bülbül Baskan E, Bradley MS, et al. Efficacy and safety of omalizumab in patients with chronic idiopathic/spontaneous urticaria who remain symptomatic on $\mathrm{H} 1$ antihistamines: a randomized, placebo-controlled study. J Invest Dermatol. 2015;135(1):67-75. doi: 10.1038/jid.2014.306.

14. Maurer M, Rosén K, Hsieh HJ, Saini S, Grattan C, Gimenéz-Arnau A, et al. Omalizumab for the treatment of chronic idiopathic or spontaneous urticaria. N Engl J Med. 2013;368(10):924-35. doi: 10.1056/NEJMoa1215372.

15. Kaplan A, Ledford D, Ashby M, Canvin J, Zazzali JL, Conner E, et al. Omalizumab in patients with symptomatic chronic idiopathic/spontaneous urticaria despite standard combination therapy. J Allergy Clin Immunol. 2013;132(1):101-9. doi: 10.1016/j.jaci.2013.05.013.

16. Sanclemente G, Burgos C, Nova J, Hernández F, González C, Reyes MI, et al. The impact of skin diseases on quality of life: A multicenter study. Actas Dermosifiliogr. 2017;108(3):244-52. doi: 10.1016/j. ad.2016.11.008. 


\section{ANEXO 1}

\section{Encuesta sobre urticaria}

Estimado doctor: agradecemos que responda la siguiente encuesta, la cual se realiza de manera anónima, para conocer sus preferencias en cuanto al tratamiento y diagnóstico de la urticaria.

1. Es usted:
a. Residente de dermatología
b. Especialista en dermatología
c. Otro profesional de la salud

2. Es usted residente de dermatología:
a. Primer año
b. Segundo año
c. Tercer año

3. Obtuvo su grado de dermatólogo hace:
a. $\quad$ Menos de 5 años
b. $\quad 5$ a 10 años
c. $\quad 10$ a 15 años
d. Más de 15 años

4. ¿En qué ciudad ejerce o estudia dermatología?

5. Usted trabaja en:
a. Hospital universitario
b. Consultorio privado
c. Consulta institucional para asegu- radores
d. Varios de los anteriores

7. En cuanto a la frecuencia de la urticaria cró nica, se presenta en la población general:
a. $\quad$ Menos del $1 \%$ b. $2 \%-3 \%$
c. $\quad 5 \%$
d. $\quad$ Hasta el $10 \%$

8. ¿Cuál es la frecuencia de urticaria crónica en su consulta?:
a. $\quad$ Menos del $5 \%$ b. Del $5 \%$ al $10 \%$
c. Más del $10 \%$

9. De ese porcentaje, ¿qué proporción se debe a urticaria crónica espontánea (no dermogra fismo ni otras urticarias físicas)?:
a. Más del $50 \%$
b. $\quad$ Entre el $20 \%$ y el $50 \%$
c. Menos del $20 \%$

10. Ante un paciente con urticaria crónica, usted rutinariamente solicita:
a. Ningún paraclínico
b. Cuadro hemático, pruebas funcio- nales tiroideas y coprológico
c. Cuadro hemático, pruebas funcio- nales tiroideas, prueba de hepatitis y búsqueda de Helicobacter pylori
d. Cuadro hemático, pruebas funcio- nales tiroideas e inmunoglobulina $\mathrm{E}$

11. ¿Practica usted de rutina una biopsia de piel en los pacientes con urticaria crónica?:
a. Sí
b. No

12. ¿Practica usted una prueba de provocación en pacientes con urticaria física?:
a. Sí
b. No 
13. En cuanto a la medición de la gravedad UAS (escala de actividad de la urticaria) y UAS7:
a. No conoce estas escalas
b. Conoce las escalas, pero no las mide de rutina
c. Ocasionalmente las utiliza
d. Siempre las utiliza

14. ¿Realiza usted la medición de escalas de calidad de vida para urticaria crónica, como, por ejemplo, el CU-Q20L (cuestionario de la calidad de vida en urticaria crónica)?:
a. No conoce estas escalas
b. Conoce las escalas, pero no las mide de rutina
c. Ocasionalmente las utiliza
d. Siempre las utiliza

15. ¿Cómo es el organigrama de tratamiento que usted utiliza en los pacientes con urticaria crónica espontánea?:

a. Antihistamínico anti-H1 de primera generación en dosis usual LUEGO adición antihistamínico anti- $\mathrm{H}_{2}$ LUEGO adición de antileucotrieno

b. Antihistamínico anti-H1 de segunda generación en dosis usual LUEGO adición de otro antihistamínico anti$\mathrm{H} 1$ de segunda generación en dosis usual

c. Antihistamínico anti-H1 de segunda generación hasta 4 veces la dosis LUEGO adición de antileucotrieno o ciclosporina

d. Antihistamínico anti-H1 de segunda generación en dosis hasta 4 veces dosis LUEGO adición de omalizumab
16. Sobre la calidad de vida de los pacientes con urticaria crónica, usted considera:
a. El impacto de la enfermedad es mínimo sobre la calidad de vida
b. Se afectan de manera relevante la calidad del sueño y la productividad laboral
c. No es tan relevante, pues es mayor el impacto de otras condiciones dermatológicas como la psoriasis
d. Desconozco la existencia de estu- dios orientados a evaluar la relación entre urticaria crónica y calidad de vida

17. Sobre la coexistencia de angioedema con episodios de urticaria crónica, usted considera que:

a. Es muy poco frecuente, de manera que no es un evento relevante en las decisiones terapéuticas

b. Puede ocurrir en el 50 \% o más de los pacientes con urticaria crónica espontánea

c. Son condiciones independientes y tal coexistencia no está demostrada

d. Desconozco los estudios orientados a identificar la frecuencia de la coexistencia de estas condiciones 